\title{
Terahertz Imaging Progress at Capital Normal University
}

\author{
Guozhong Zhao ${ }^{1,2,3,}$, Jiayi Yu ${ }^{1,2,3}$, Yashang $\mathrm{Li}^{1,2,3}$, Jia Wang ${ }^{1,2,3}$ \\ ${ }^{1}$ Department of Physics, Capital Normal University, Beijing, China \\ ${ }^{2}$ Beijing Key Lab of Terahertz Spectroscopy and Imaging, Key Lab of Terahertz Optoelectronics, Ministry of Education, Beijing, China \\ ${ }^{3}$ Beijing Advanced Innovation Centre of Imaging Technology, Beijing, China
}

\author{
Email address: \\ Guozhong-zhao@cnu.edu.cn (Guozhong Zhao) \\ *Corresponding author
}

\section{To cite this article:}

Guozhong Zhao, Jiayi Yu, Yashang Li, Jia Wang. Terahertz Imaging Progress at Capital Normal University. International Journal of Astrophysics and Space Science. Vol. 5, No. 5, 2017, pp. 79-84. doi: 10.11648/j.ijass.20170505.12

Received: August 1, 2017; Accepted: October 28, 2017; Published: December 1, 2017

\begin{abstract}
The progress on terahertz imaging at Capital Normal University in Beijing is presented. Our works on Terahertz Imaging include the active and passive imaging. For the active terahertz imaging, the pulse and continue wave terahertz imaging are studied, respectively. The active terahertz pulse imaging is based on the terahertz time-domain spectroscopy (THz-TDS). A practical focus-plane imaging system is built up based on the THz-TDS with the probe-beam-expanded electro-optical sampling and an infrared CCD imaging. The active terahertz continuous wave imaging is based on a $\mathrm{CO}_{2}$-laser-pumped terahertz coherent source and an uncooled bolometer array of terahertz camera. The active terahertz polarization imaging and wave front imaging is developed. For the passive terahertz imaging, the low frequency of radiometers are used to detect the point-to-point beam-scanned terahertz signal by the optical focusing and scanning system so that the passive $\mathrm{THz}$ imaging is realized for the security inspection of human body. The related components and image processing methods are studied and used for the improvement of imaging speed and resolution. The research on $\mathrm{THz}$ imaging technology at Capital Normal University is opening up a potential application in the field of non-destructive testing and security inspection for the terahertz technology.
\end{abstract}

Keywords: Terahertz, Active, Passive, Imaging, Non-destructive Testing, Security Inspection

\section{Introduction}

Terahertz (THz) imaging is a new hot topic in the current field of imaging technology [1-5]. THz imaging has the advantage to penetrate most of non-metal and non-polar materials for the detection of concealed objects, while it is harmless to biological tissue and human body [6-14]. THz imaging can be divided into two types: active $\mathrm{THz}$ imaging and passive $\mathrm{THz}$ imaging. According to the difference of $\mathrm{THz}$ source, the active $\mathrm{THz}$ imaging can be divided into the pulse $\mathrm{THz}$ imaging and continuous wave $\mathrm{THz}$ imaging. Generally, THz image is extracted from the intensity distribution of $\mathrm{THz}$ signal. The active $\mathrm{THz}$ pulse imaging is developed based on the $\mathrm{THz}$ time-domain spectroscopy [1], which is based on the amplitude distribution of $\mathrm{THz}$ transmission. At the same time, the polarization or phase distribution of $\mathrm{THz}$ signal as the different information sources are investigated. Nick C. J. van der Valk et al presented a new method to measure the polarization state of a terahertz pulse by using a modified electro-optic sampling setup [2]. Many variations of this scheme have been presented since then. Most of them were based on the pulsed $\mathrm{THz}$ time-domain spectroscopic imaging [3-6]. Pallavi Doradla et al presented some reflection measurements of 5-mm-thick sections of colorectal tissues using a polarization-specific detection technique based on continuous wave $(\mathrm{CW})$ terahertz source [7]. $\mathrm{THz}$ polarization imaging provides more information on the measured targets. $\mathrm{THz}$ polarization imaging based on continuous wave $(\mathrm{CW})$ terahertz source is studied in our laboratory recently [15]. The higher resolution of terahertz imaging is achieved at 3.1 $\mathrm{THz}$ of operating frequency. However, the imaging distance of the high frequency, such as $3.1 \mathrm{THz}$, of terahertz imaging is limited by $\mathrm{THz}$ attenuation due to the water vapor absorption. Moreover, the focal plane terahertz imaging is developed to obtain much more frequency domain of spectral information [16-19]. The focal plane imaging can be realized as a quasi-near field imaging so that it can obtain a higher 
resolution. However, the view field of focal plane imaging is limited due to the size of electro-optic crystal. In recent years, the passive terahertz imaging is developed for the longer imaging distance and the larger imaging view field [20]. The sensitivity of terahertz radiometer is a key factor for the contract and resolution of passive terahertz imaging. Compared with the visible light and infrared rays, the research and application of terahertz polarization and wave front imaging are still underdeveloped so far.

In this paper, the active $\mathrm{THz}$ wave front imaging is studied based on the continuous wave $\mathrm{THz}$ source. At the same time, the performance of passive $\mathrm{THz}$ imaging is improved by the fast opto-mechanic scanning and higher frequency and array of $\mathrm{THz}$ detection. The development of these $\mathrm{THz}$ imaging technology is an important research direction at $\mathrm{THz}$ laboratory of Capital Normal University. These researches are helpful for the public security inspection and $\mathrm{THz}$ non-destructive testing.

\section{Active Terahertz Imaging}

\subsection{Overview of Active Terahertz Imaging}

The active terahertz pulse imaging based on the terahertz time domain spectroscopy (THz-TDS) has been investigated in our laboratory for some years [21-25], including $\mathrm{THz}$ transmission/amplitude imaging [21], $\mathrm{THz}$ polarization imaging [22-23], and THz phase imaging [24-25]. At the same time, the active $\mathrm{THz}$ pulse imaging is developed and applied for the quasi-near field measurement based on the terahertz time-domain spectroscopy with the probe-beam-expanded femtosecond pulse laser and an infrared CCD detection [16-19].

The active $\mathrm{THz}$ imaging based on a continuous wave $\mathrm{THz}$ source and a terahertz camera is studied in our group [15]. The terahertz imaging system consists of a $\mathrm{CO} 2$-pumping gas $\mathrm{THz}$ laser SIFIR50 and a NEC camera of array detector IRV-T0831C, shown as figure 1. The parabolic mirrors M1, $\mathrm{M} 2$, and M3 are used to calibrate and focus the terahertz beam. Two metal-grid polarizers $\mathrm{P} 1$ and $\mathrm{P} 2$ are used to control and measure the polarization of terahertz beam. The experimental results show that the active $\mathrm{THz}$ polarization imaging has a high spatial resolution [15]. Recently, we developed $\mathrm{THz}$ wave front imaging by using the Hartmann plate instead of the polarizer P2 based on the same system above.

\subsection{New Progress of Active Terahertz Imaging}

The performance design of $\mathrm{THz}$ imaging system is a balance depended on its application. The active $\mathrm{THz}$ imaging based on the THz-TDS system can take an important role in the aspects of matter identification and non-destructive inspection. However, the imaging speed and resolution of active THz pulse imaging based on THz-TDS is limited by the point-to-point mechanical scanning, especially, the imaging view field or image size cannot be too large. $\mathrm{THz}$ polarization imaging can provide more information with the different polarization parameters. At the same time, as one of the important electromagnetic wave parameters, the phase distribution of $\mathrm{THz}$ wave can also provide special information on $\mathrm{THz}$ image. Based on the THz-TDS imaging, we have demonstrated the $\mathrm{THz}$ phase imaging can provide more information on the $\mathrm{THz}$ wave penetrating samples.

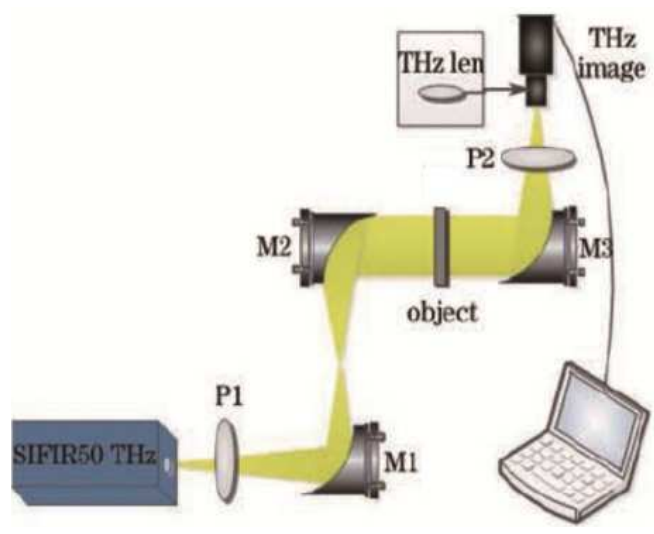

Figure 1. Schematic setup of active terahertz imaging.

The active $\mathrm{THz}$ wave front imaging based on the continuous wave $\mathrm{THz}$ source and the $\mathrm{THz}$ camera is studied by adding a Hartmann plate on the front of a NEC THz camera. The imaging system is same as shown as figure 1, just change the polarizer P2 into a Hartmann plate. The Hartman plate and the camera form into a Hartmann sensor which can detect the wave front distribution of $\mathrm{THz}$ wave through the object imaged by extracting the phase information from the intensity image. In fact, the wave front imaging present the information of phase contrast to detect and display the phase distribution of electromagnetic wave carrying with the inner characteristic of detected object.

The extracting of phase information for the wave front imaging include three steps. Firstly, the images of spot intensity distribution of $\mathrm{THz}$ wave through the object and the reference without through the object are acquired, respectively, by the Hartmann sensor. Compared with the reference image, the spot image of $\mathrm{THz}$ wave through the object with the inner structure show some distortion. Then the spot center coordinates of reference and object are calculated, respectively. The slope of distorted spot relative to the reference spot can be obtained from the difference of the spot center coordinates. Finally, the wave front distribution is obtained by means of the zonal estimation in the wave front reconstruction theory [26]. In detail, the center coordinates of distorted spots can be calculated as follows

$$
\begin{array}{r}
\bar{x}=\frac{\sum_{i=1}^{L}\left[\sum_{j=1}^{M} I(i, j) \cdot x_{i}\right]}{\sum_{i=1}^{L}\left[\sum_{j=1}^{M} I(i, j)\right]} \\
\bar{y}=\frac{\sum_{i=1}^{L}\left[\sum_{j=1}^{M} I(i, j) \cdot y_{i}\right]}{\sum_{i=1}^{L}\left[\sum_{j=1}^{M} I(i, j)\right]}
\end{array}
$$


Where $\mathrm{x}_{\mathrm{i}}$ and $\mathrm{y}_{\mathrm{i}}$ are the $\mathrm{x}$ - and $\mathrm{y}$-direction coordinates of ( $\mathrm{i}$, j) pixel point on the detection area of NEC camera, respectively, $I(i, j)$ is the intensity on $(i, j)$ pixel. $L$ and $M$ are the $\mathrm{x}$ - and $\mathrm{y}$-direction of pixel numbers in the single detection window. Comparing the center coordinate of distorted spot with the reference coordinate $\left(\mathrm{x}_{0}, \mathrm{y}_{0}\right)$, the offset $\Delta \mathrm{x}$ and $\Delta \mathrm{y}$ can be obtained as follows

$$
\Delta \mathrm{x}=\bar{x}-x_{0}, \Delta y=\bar{y}-y_{0}
$$

If the distance of Hartmann plate to detection area of camera is $d$, then the slope of wave front of each spot along the $\mathrm{x}$ - and $\mathrm{y}$-direction is as follow

$$
S_{x}=\frac{\Delta x}{d}, S_{y}=\frac{\Delta y}{d}
$$

Further the relation between the slope and the phase of grid mode can be given by Southwell [17]

$$
\emptyset^{i, j}=\frac{1}{4}\left(\emptyset^{i-1, j}+\emptyset^{i, j-1}+\emptyset^{i+1, j}+\emptyset^{i, j+1}\right)+\frac{h}{8}\left\{\left(S_{x}^{i, j-1}+S_{x}^{i, j}\right)-\left(S_{x}^{i, j+1}+S_{x}^{i, j}\right)\right\}+\frac{h}{8}\left\{\left(S_{y}^{i-1, j}+S_{y}^{i, j}\right)-\left(S_{y}^{i+1, j}+S_{y}^{i, j}\right)\right\}
$$

On the basis of this formula, the phase of each spot can be obtained by the iterative calculation.

In the experiment of wave front imaging, the object imaged is placed into the parallel $\mathrm{THz}$ beam. The Hartmann plate is used in front of THz lens and NEC THz camera instead of the polarizer $\mathrm{P} 2$ in figure $1 . \mathrm{THz}$ source SIFIR50 output $\mathrm{THz}$ beam with the average power about $15 \mathrm{~mW}$ at $3.11 \mathrm{THz}$ of operating frequency. The uncooled bolometer array of camera IRV-S830C with $320 \times 240$ pixels and the pixel pitch of 23.5 $\mu \mathrm{m}$, which operating frequency is $1-7 \mathrm{THz}$, is used to acquire the image of $\mathrm{THz}$ bean. The Hartmann plate consists of a set of aperture array on the $0.2 \mathrm{~mm}$ thickness of stainless steel sheet with the aperture diameter of $1 \mathrm{~mm}$ and the square array period of $2 \mathrm{~mm}$. As a testing, a wedge of Teflon is used as the object imaged. The acquired images of reference spot and distorted spot are shown in figure 2, where the red and blue spots are the images of Hartmann aperture centers with and without the wedge Teflon in the THz path. The area shown in figure 2 is a part of Hartmann plate.

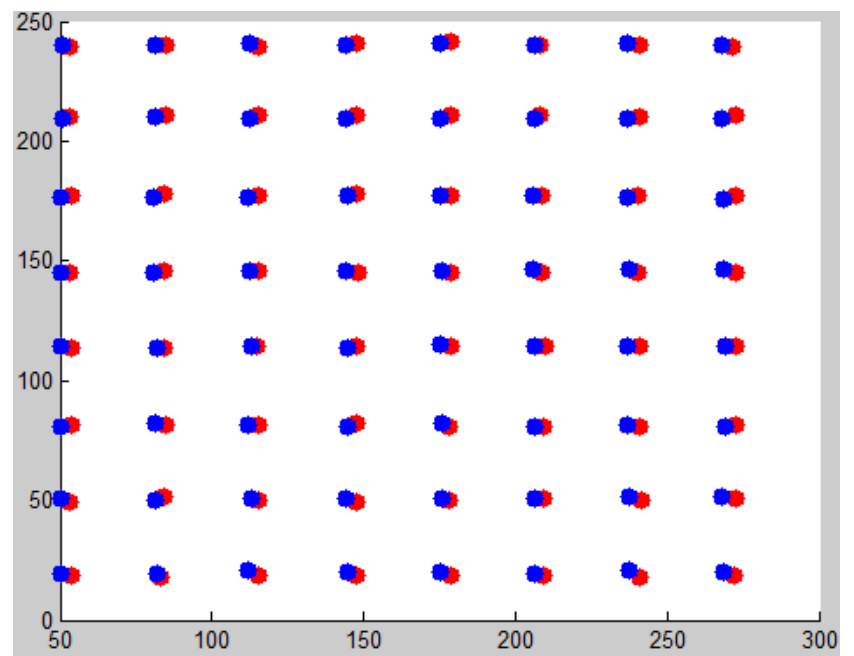

Figure 2. Images of spot center with and without the wedge Teflon in $\mathrm{THz}$ beam, blue spot is the reference.
It can be seen that the spot center after the wedge Teflon plate deviated from the reference position and the offset increased with increasing of Teflon thickness. By extracting the phase of $\mathrm{THz}$ beam at each spot, the wave front profile can be obtained as shown as figure 3 .

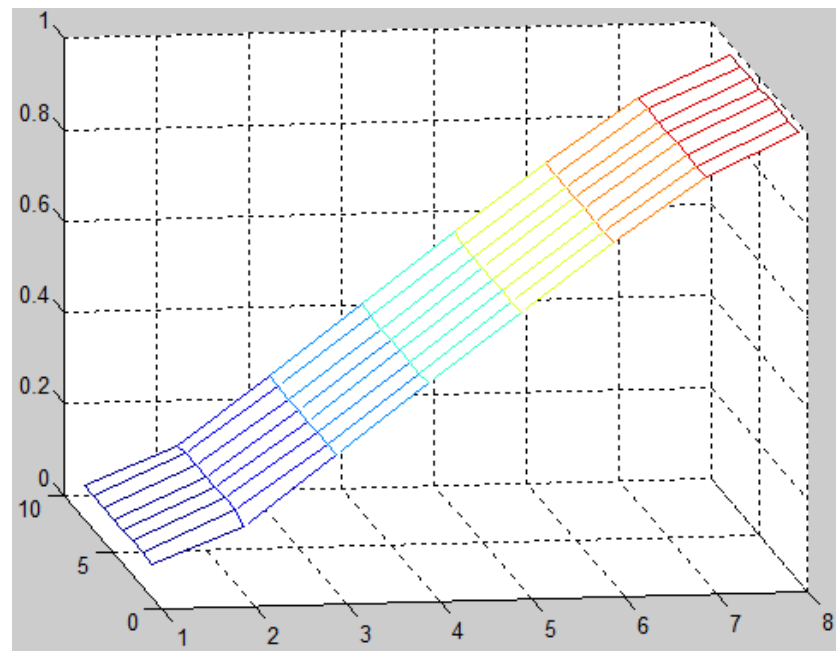

Figure 3. Wave front profile of wedge Teflon plate.

Figure 3 shows that the phase difference at two ends of wedge plate is 0.924 rad. By taking account of the amplification of view field to 1.4 times by $\mathrm{THz}$ lens, the measured real phase difference should be $0.66 \mathrm{rad}$ which corresponding to the path difference about $10 \mu \mathrm{m}$. The measured result agree well with the slope of wedge Teflon plate. Therefore, the wave front imaging can not only see the profile of objects, but also measure the size parameters of objects imaged. It is not difficult to understand that the wave front imaging is possible to detect the defects hidden inside the objects, and even to measure their size. THz wave front imaging has potential application in the field of non-destructive testing. 


\section{Passive Terahertz Imaging}

\subsection{Overview of Passive Terahertz Imaging}

The passive terahertz imaging system usually use the heterodyne terahertz receivers to detect $\mathrm{THz}$ signal from the object imaged. For the security inspection of human body and the objects they carried, $\mathrm{THz}$ radiation is so week that the current $\mathrm{THz}$ camera is difficult to acquire any $\mathrm{THz}$ images due to the poor sensitivity of these camera. On the other hand, $\mathrm{THz}$ array detector with the high operating frequency such as 1 $\mathrm{THz}$ above and the more pixel number such as more than $640 \times 480$ pixels is expansive or unavailable for the common users. So far, most of the passive millimeter wave imaging system or so called $\mathrm{THz}$ passive imaging system is operating at the low frequency below $300 \mathrm{GHz}$, such as $91 \mathrm{GHz}$ [26], $100 \mathrm{GHz}$ [27], $140 \mathrm{GHz}$ [28], $220 \mathrm{GHz}$ [29], $345 \mathrm{GHz}$ [30], and so on. Due to the lack of $\mathrm{THz}$ array detector or camera with high sensitivity, the passive $\mathrm{THz}$ imaging system uses the optical scanning system to acquire $\mathrm{THz}$ signal at the local position of object imaged, and normally operate at a single frequency. Correspondingly, the imaging speed of optical scanning system is limited. The imaging distance or view field of passive $\mathrm{THz}$ imaging system is also limited by the poor sensitivity of $\mathrm{THz}$ receiver. Therefore, the passive $\mathrm{THz}$ imaging is developing on its road. Many groups are still working on this field now.

The passive terahertz imaging is developed in our group for many years based on the heterodyne terahertz receivers with the different operating frequencies and the beam-scanned imaging system [20]. The four main research aspects for the building of this system include the optical scanning system, the terahertz wave focusing and receiving system, the measurement and control system based on the electronics, and the image processing software. The different imaging system with the different imaging distance, imaging speed, and resolution is studied and developed for the different aim of applications. For example, the imaging distances of $1 \mathrm{~m}, 2 \mathrm{~m}$, $5 \mathrm{~m}$, and $10 \mathrm{~m}$ are realized by the different diameter of focusing reflectors. At the same time, the imaging speed is improved step by step. The imaging speed of one frame per second is achieved recently [20]. Now a passive THz imaging system with the larger view field, the faster imaging speed, and the higher resolution is gradually being developed and improved.

\subsection{New Progress of Passive Terahertz Imaging}

As described above, the passive terahertz imaging system is based on a heterodyne receiver and a beam-scanned and focusing optical system with the fast rotating mirrors and a focusing reflector. It focus the terahertz radiation from the source, such as a human body, onto a heterodyne terahertz receiver. So the terahertz radiation is point-by-point scanned and focused to the receiver. A polyethylene window, which transmission is around $80 \%$ in terahertz frequency range we used, is used for the penetration of terahertz wave and the protection of optical units. A visible light of CCD is integrated into this system for the identification of terahertz image in the photos.

The used receiver is a typical millimeter wave radiometer with a mixer and a local oscillator together with a low-noise amplifier. This kind of heterodyne receiver has the high sensitivity and can operate at the room temperature [20]. However, the high frequency of heterodyne receiver, especially the array of these receivers, is expansive or unavailable. We have to develop the optical scanning system and do the passive imaging by the single pixel or less pixels of detection.

In order to improve the imaging speed, a four-channel heterodyne terahertz receiver operating at the frequency of $250 \mathrm{GHz}$ is developed and integrated into our passive terahertz imaging system. At the same time, the optical scanning and focusing system is improved by designing the combination of fast rotating ellipsoid mirrors and a plane reflector to increase the scanning speed. The imaging speed of one frame per second is realized on our prototype [20].

By the experimental measurement, it is determined that this terahertz imaging system can work on a $1.7 \mathrm{~m}$ to $6 \mathrm{~m}$ of imaging distance by adjusting its focal length of focusing reflector and the position of terahertz receivers. The spatial resolution of this passive terahertz imaging system achieve at least $2 \mathrm{~cm}$, seen in figure 4 . It can get one frame of terahertz images with the different frequencies during one second of time.
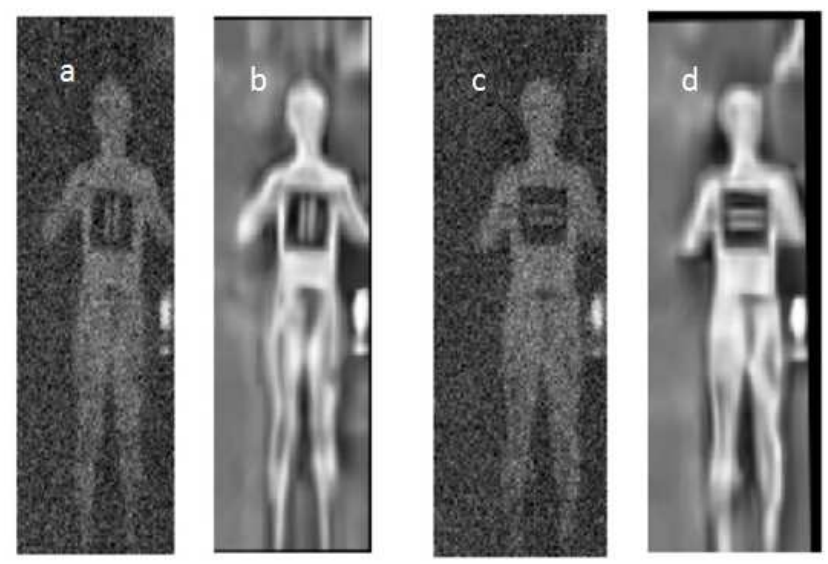

(a) original y- (b) processing $y-(c)$ original $x-(d)$ processing $x$-images.

Figure 4. Resolution of $\mathrm{THz}$ images from the passive $\mathrm{THz}$ imaging system with image processing.

In the experiment, several objects like a mobile phone, a metallic box, and a leather measuring tape, are placed inside the cloth of human body. Their shape and size can be clearly seen in the terahertz images. As a result, it is easy to match the found objects in the terahertz images with the visible photo. So it provides an important tool to recognize the hidden targets carried by the human body using the terahertz imaging.

Moreover, the different frequencies of terahertz receivers are integrated into our system for seeing and distinguishing the image difference of different objects which shield $\mathrm{THz}$ beam from the human body. The image processing and fusion 
are investigated and applied to our software of $\mathrm{THz}$ passive imaging system [31]. It is also integrated into the same interface of program within the different frequencies of terahertz images together with their image fusion. The terahertz images can be displayed on the screen of computer together with the parameters setting of imaging system including the original images, the processed image, the fusion of terahertz images with the different frequencies, and the terahertz images in the visible light of photo. The terahertz images can also be identified in the visible-light of photo, seen as figure 5 .

It is worthy to mention that the passive terahertz imaging is potential for the imaging of long distance and large field of view. With the characteristic of penetration and harmless of terahertz radiation, it is possible to apply the passive terahertz imaging into the security inspection and non-destructive testing.
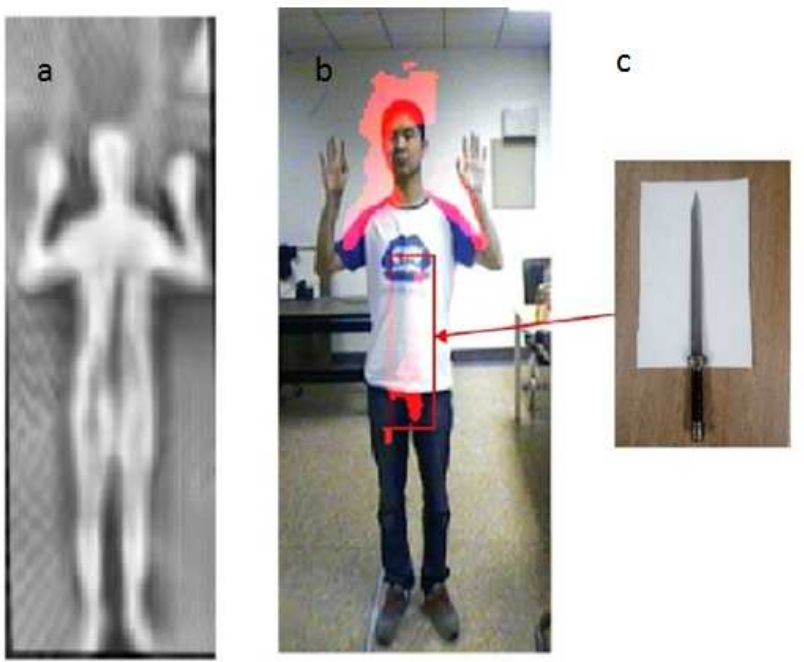

(a)THz image (b) photo of human body with knife (c) hidden knife.

Figure 5. Hidden object identified by THz images.

\section{Summary}

In summary, the active and passive terahertz imaging are investigated and developed at Capital Normal University. The active terahertz wave front imaging is sensitive to detect the hidden objects and even their size. The passive terahertz imaging is potential for the long distance and large field-view of imaging. The advantage and disadvantage of different terahertz imaging technology can be seen and compared. It depends on the different needs of application, too. The further investigations and development of terahertz imaging technology are being carried out in the terahertz group of Capital Normal University.

\section{Acknowledgements}

This work is supported by National Natural Science Foundation of China with the Grants No. 61575130 and 50971094, the Scientific Research Project of Beijing Municipal Commission of Education, and Beijing Natural Science Foundation with Grant No.KZ201310028032.

\section{References}

[1] Lee Yun-Shik. "Principles of terahertz science and technology". NY: Springer, 2008.

[2] Van der Valk N. C. J., van der Marel W. A. M., Planken P., "Terahertz polarization imaging," Opt. Lett., 30 (20), 2802-2804 (2005).

[3] Zhang L. L., Zhong H., Deng C. et al, "Terahertz Polarization Imaging with Birefringent Materials," Opt. Comm., 283 (24), 4993-4995 (2010).

[4] Wang X., Cui Y., Sun W. et al, "Terahertz polarization real-time imaging based on balanced electro-optic detection," J. Opt. Soc. Am. A, 27 (11), 2387-2393 (2010).

[5] Zhang L. L., Zhong H., Deng C. et al, "THz wave polarization-controlled spectroscopic imaging for anisotropic materials," Opt. Comm., 284 (19), 4356-4359 (2011).

[6] Katletz Stefan, Pfleger Michael, Pühringer Harald, "Polarization sensitive terahertz imaging: detection of birefringence and optical axis," Opt. Express, 20 (21), 23025-23035 (2012).

[7] Doradla P., Alavi K., Joseph C., and Giles R., "Detection of colon cancer by continuous-wave terahertz polarization imaging technique," J. Biom. Opt., 18 (9), 090504-3 (2013).

[8] Yao Rui, Ding Shaohui, Li Qi, and Wang Qi, "Improvement of 2. $52 \mathrm{THz}$ Array Transmission Imaging System and Resolution Analysis," Chinese Journal of Lasers, 38 (1), 0111001-6 (2011).

[9] Luis Femando Rojas-Ochoa, David Lacoste et al, "Depolarization of backscattered linearly polarized light," J. Opt. Soc. Am. A., 21 (9), 1799-1804 (2004).

[10] Preda M., Scarlat E. I., Preda L., "Depolarization analysis of a Laser beam scattered by a dusty plasma," Proc. SPIE 5581, 509-516 (2004).

[11] Fei Liu, Xiaopeng Shao, Pingli Han, "A Novel IR Polarization Imaging System Designed by a Four-Camera Array," Proc. Of SPIE, 9124, 912419-6 (2014).

[12] Xu Canjun, Zhao Jinsong, Cai Yi et al, "Several schemes of infrared polarization imaging technology," Infrared Technology, 31 (5), 262-266 (2009).

[13] Hartwick T. S., Hodges D. T., Barker D. H., and Foote F. B., "Far infrared imagery," Appl. Opt. 15 (8), 1919-1922 (1976).

[14] Rui Yao, Qi Li, Qi Wang, "1.63-THz Transmission Imaging Experiment by Use of a Pyroelectric Camera Array," Proc. Of SPIE, 7277, 72770D (2009).

[15] Ying Liu, Yanchun Shen, Guozhong Zhao, "Terahertz polarization imaging based on the continuous wave terahertz radiations", Proc. of SPIE, 9622, 962209 (2015).

[16] Xinke Wang, Ye Cui, Dan Hu, Wenfeng Sun, Jiasheng Ye, and Yan Zhang, "Terahertz quasi-near-field real-time imaging”, Optics Communications, $2824683-4687$ (2009).

[17] Xinke Wang, Ye Cui, Wenfeng Sun, Jiasheng Ye and Yan Zhang, "Terahertz polarization real-time imaging based on balanced electro-optic detection", J. Opt. Soc. Am. A, 27 2387-2393 (2010). 
[18] Xinke Wang, Ye Cui, Wenfeng Sun, Jiasheng Ye and Yan Zhang, "Terahertz real-time imaging with balanced electro-optic detection", Optics Communications, 283 46264632 (2010).

[19] Zhenwei Xie, Xinke Wang, Jiasheng Ye, Shengfei Feng, Wenfeng Sun, Tahsin Akalin, and Yan Zhang, "Spatial terahertz modulator", Scientific Reports, 33347 (2013).

[20] Guozhong Zhao, Yanchun Shen, Jia Wang, and Jiayi Yu, New Progress of Active and Passive Terahertz Imaging, Proc. of SPIE, 10030, 100300Q (2016).

[21] Liangliang Zhang, Nick Karpowicz, Cunlin Zhang, Yuejin Zhao and X.-C. Zhang, "Real-time nondestructive imaging with terahertz waves", Optics Communications 281, 1473 (2008).

[22] Liangliang Zhang, Hua Zhong, Chao Deng, Cunlin Zhang and Yuejin Zhao, "Terahertz wave polarization analyzer using birefringent materials", Optics Express 17, 20266 (2009).

[23] Liangliang Zhang, Hua Zhong, Chao Deng, Cunlin Zhang and Yuejin Zhao, "Characterization of birefringent material using polarization-controlled spectroscopy", Optics Express 18, 20491 (2010).

[24] Liangliang Zhang, Yan Zhang, Cunlin Zhang, Yuejin Zhao and Xiaohua Liu, "Terahertz multiwavelength phase imaging without $2 \pi$ ambiguity", Optics Letters 31, 3668 (2006).

[25] Liangliang Zhang, Hua Zhong, Yan Zhang, Nick karpowizc,
Cunlin Zhang, Yuejin Zhao and X.-C. Zhang, "Terahertz wave focal-plane multiwavelength phase imaging", Journal of the Optical Society of America A 25, 1187 (2009).

[26] Aleksandar Đuric, Andreas Magun, Axel Murk, Christian Mätzler, and Niklaus Kämpfer, The Fully Polarimetric Imaging Radiometer SPIRA at $91 \mathrm{GHz}$, IEEE Transactions on Geoscience and remote sensing, 46 (8), 2323-2336 (2008).

[27] Appleby R, Wallace H, Standoff detection of weapons and contrab and in $100 \mathrm{GHz}$ to $1 \mathrm{THz}$ region, IEEE Transactions on Antenna and Propogation, 55, 2944-2956 (2007).

[28] Toshihiko K, Hiroki S, Hideaki M, Koichi M, Mitsunori N, Hiroshi S, and Kraisorn T, "A 140-GHz Quad-Receivers IC and Sub-Assembly for Compact Passive Imaging Sensors", Microwave Symposium Digest, 1-3 (2011).

[29] Stanko S, Wirtz S, "Millimeter wave imaging for concealed weapon detection and surveillance at up to $220 \mathrm{GHz}$ ", Proc. Of SPIE, 6948, 69480N (2008).

[30] Gropp C, Kulesa G, Lichtenberger A, Weinreb S, A progress update on SuperCam, "A $345 \mathrm{GHz} 64$ pixel heterodyne imaging spectrometer", in Proceedings of 22th intemational Symposium on Space Terahertz Technology, Tucson, 2011, $\mathrm{p} 15$.

[31] Li Tian, Yanchun Shen, Weiqi Jin, Guozhong Zhao, and Yi Cai, "Processing and fusion of passively acquired, millimeter and terahertz images of the human body", Opt. Eng. 56 (12), 111-222 (2017). 